\title{
Repetitive peripheral magnetic stimulation improves severe upper limb paresis in early acute phase stroke survivors
}

\author{
Shigeru Obayashi* and Rina Takahashi \\ Department of Rehabilitation Medicine, Dokkyo Medical University Saitama Medical Center, Saitama, Japan
}

\begin{abstract}
.
BACKGROUND: It is very difficult for patients with severe upper extremity (UE) paresis after stroke to achieve full recovery because of the lack of a definitive approach for improving severe UE paresis immediately after onset.

OBJECTIVE: to investigate the effects of repetitive peripheral magnetic stimulation (rPMS) on severe UE paresis during early acute phase of stroke.

METHODS: Nineteen participants with severe UE disability met the criteria. 10 subjects received 15-20 minutes of rPMS prior to standard care per session, while 9 age- and severity-matched subjects received two times 20 minutes of standard care. Outcome measures included UE motor section of the Fugl-Meyer Motor Assessment Scale (FMA-UE), Wolf motor function test (WMFT), and box and block test (BBT).

RESULTS: The rPMS group received treatment (average sessions: 7.8) after a median 9.2 days from stroke (16.5 sessions after 5 days for control). To adjust the different treatment durations, we defined "progress rate" as the gains of UE function scores divided by treatment duration. The progress rate was significantly different in FMA-UE and WMFT, but not in BBT. CONCLUSIONS: The present study suggested beneficial effects of rPMS on severe UE paresis during early acute phase of stroke.
\end{abstract}

Keywords: Cortical reorganization, peripheral magnetic stimulation, motor recovery, stroke, upper extremity

\section{Abbreviations}

BBT

box and block test

ECR extensor carpi radialis

EDC extensor digitorum communis

EIP extensor index proprius

EMG electromyography

FAS functional ability scale

FDS flexor digitorum superficialis

FES functional electrical stimulation
FMA Fugl-Meyer Motor Assessment Scale

f-MRI functional magnetic resonance imaging

NMES neuromuscular electrical stimulation

rPMS repetitive peripheral magnetic stimulation

SC standard care

SIAS stroke impairment assessment set

UE upper extremity

WMFT Wolf motor function test
*Address for correspondence: Shigeru Obayashi, MD, PhD, Department of Rehabilitation Medicine, Dokkyo Medical University Saitama Medical Center, 2-1-50 Minami-Koshigaya, Koshigaya, Saitama, 343-8555, Japan. Tel.: +48965 1111; E-mail: ohbayash@dokkyomed.ac.jp.

\section{Introduction}

Upper extremity (UE) paresis after stroke disturbs functional independence. Only a few patients 
with severe UE paresis have reportedly achieved full recovery (Nakajima et al., 1994), mainly because of the lack of a definitive approach to improving severe UE paresis immediately after stroke.

Recently, there has been increasing evidence that functional electronic stimulation (FES), such as neuromuscular electrical stimulation (NMES), may facilitate motor restoration of UE function in chronic phase of stroke (Carda et al., 2017; Cauraugh, Light, Kim, Thigpen, \& Behrman, 2000; Hara, Obayashi, Tsujiuchi, \& Muraoka, 2013). On the other hand, the superiority of NMES to standard care still remains controversial (Wilson et al., 2016), perhaps partly because of a lack of justification for specific treatment parameters (Whitall, 2004). Furthermore, uncertain effects of NMES may be due to the placement of electrodes on stimulated muscles limited to wrist and hand only (Chae et al., 1998; Francisco et al., 1998; Powell, Pandyan, Granat, Cameron, \& Stott, 1999).

Like NMES, repetitive peripheral magnetic stimulation (rPMS) stimulates action potential in motor axons evoking muscle contraction, and is therefore expected to become another neuromodulator facilitating motor recovery after stroke. The definite advantage of rPMS over NMES is that rPMS can penetrate into deeper regions of muscles without pain. So far, however, although some studies have demonstrated that rPMS is effective for spasticity after stroke, there has been no literature to demonstrate the efficacy of rPMS on upper extremity paresis despite some efforts using various parameters (Barker, 1991; Beaulieu \& Schneider, 2013; Krewer, Hartl, Muller, \& Koenig, 2014; Struppler, Angerer, Gundisch, \& Havel, 2004; Struppler et al., 2007; Struppler, Havel, \& Muller-Barna, 2003).

On the other hand, previous studies have suggested that the greatest gain of recovery tends to occur immediately after stroke, with a slower pace over time (Kwakkel, Kollen, \& Twisk, 2006; Kwakkel, Kollen, van der Grond, \& Prevo, 2003). These results may indicate the benefit of early intervention by rPMS or NMES during the very early acute phase of stroke. In fact, we recently demonstrated the favorable efficiency of coupled EMG-triggered NMES and cyclic NMES on severe UE function for early acute phase patients with stroke (Obayashi, 2020). Given that peripheral stimulation for affected muscles can help facilitate motor recovery, it is possible that rPMS is also useful for facilitating motor recovery of UE paresis.

Now, to prove this hypothesis, we investigated the effects of rPMS applied to UE muscles including shoulder, elbow, wrist and fingers on UE function during early acute phase of stroke.

\section{Methods and materials}

\subsection{Participants}

Subjects were recruited from an academic medical center from February 2019 to February 2020. Inclusion criteria were: (1) medically stable; (2) hospitalized adults within two weeks of stroke; (3) age 20-89 years; (4) intact skin on the hemiparetic arm; (5) adequate cognition to participate; (6) first-ever, severe UE paresis (stroke impairment assessment set (SIAS): knee-mouth test range 1-3; finger function test 1a-3). Exclusion criteria were: (1) past history of stroke, brain injury or brain tumor; (2) metal implant in head or within stimulation area; (3) dysphasia; (4) history of cardiac arrhythmia with hemodynamic instability; (5) comorbidity with neurodegenerative diseases and mental disorders; (6) uncontrolled seizure disorder; (7) disturbed consciousness; (8) implanted stimulator (such as cardiac pacemaker); (9) pregnancy. The study protocol was approved by the ethics committee of Dokkyo Medical University Saitama Medical Center (Registration Number:1902) and the study was conducted in accordance with relevant ethical guidelines.

\subsection{Interventions}

rPMS was applied to upper limb muscles including extensor digitorum communis (EDC), extensor carpi radialis (ECR), flexor digitorum superficialis (FDS), triceps brachii, biceps brachii, and anterior or middle head of deltoid. We used a peripheral magnetic stimulator (Pathleader, IFG, Sendai, Japan) for rPMS treatment. This round coil stimulator generated biphasic $350 \mu \mathrm{sec}$ with magnetic gradients of up to $15 \mathrm{kT} / \mathrm{sec}$, thus producing repetitive contractionrelaxation cycles that enhance proprioceptive input from target muscles. We applied an intermittent ON/OFF stimulation protocol consisting of three times 10 consecutive stimulations for each muscle per session, where one stimulation $(\mathrm{ON})$ was continued for $2 \mathrm{sec}$ with a frequency of $30 \mathrm{~Hz}$ and intensity of $70 \%$ MSO (maximal stimulator output: corresponding to 0.65 Tesla), and then the stimulation intervals (OFF period) were set at $2 \mathrm{sec}$. Deltoid, triceps brachii, ECR and EDC were mandatory target muscles for rPMS. Additional application of rPMS 
to flexor muscles (biceps brachii and FDS) was given only to patients with flaccid muscle tone. The stimulation session was conducted with patients sitting on a chair in front of a table.

\subsection{Treatment program}

The participants were automatically allocated to two groups, the rPMS group and the standard care (SC) group, each matching each other in terms of age, start from onset, and severity of UE function. This intervention group received 15-20 minutes of rPMS and subsequent 20 minutes of SC every other day, 3 days per week, or two consecutive 20 minutes of SC 2 days per week, until the patients were transferred to another intensive restorative hospital. The SC group as control condition received a two consecutive 20 minutes of SC per day, 5 times per week until the transfer period. One unique characteristic of rehabilitation medicine in acute care hospitals in Japan is that it is concluded within a restricted time period of approximately 2 to 4 weeks, and is then continued in proper restorative rehabilitation care units of other hospitals. Note that different patients had different durations of treatment (3-32 sessions) depending on the transfer period.

Outcome measures included the upper extremity (UE) motor section of the Fugl-Meyer Motor Assessment Scale (FMA) (Fugl-Meyer, Jaasko, Leyman, Olsson, \& Steglind, 1975), Wolf motor function test (WMFT), functional ability scale (FAS) (Wolf et al., 2001), and box and block test (BBT) (Desrosiers, Bravo, Hebert, Dutil, \& Mercier, 1994). These measures were assessed at pretreatment baseline and after the end of treatment. Scoring of the FMA motor section (0-66 points) requires the subjects to perform 33 movements. Their performance of each movement is scored on a 3-point ordinal scale ( 0 , cannot perform; 1 , performs partially; 2, performs fully). In WMFT sessions, all tasks are performed as quickly as possible and are truncated at 120 seconds. Tasks $1-6$ of the WMFT involve timed joint-segment movements, and tasks $7-15$ consist of timed integrative functional movements. BBT estimates gross manual dexterity. BBT consists of moving, one by one, the maximum number of blocks from one compartment of a box to another of equal size within 60 seconds.

\subsection{Progress rate}

To decide which treatment (rPMS or standard care) would be more effective for motor recovery, gains of improvement in UE function were compared. Because different patients received different durations of treatment depending on the transfer period, gains needed to be adjusted for comparison between the two treatments. Thus, progress rate was defined as gains of UE function scores divided by treatment duration and then compared between groups.

\subsection{Statistical analysis}

Statistical analysis was performed by MannWhitney $U$ test for nonparametric data using SPSS ver. 26. Results were accepted as statistically significant at $P<0.05$. Finally, effect size ( $r$ value) of outcome measures was calculated to measure the magnitude of treatment effect: $r=Z / \sqrt{ } N$, where $\mathrm{N}$ is the total number of samples. $r$ values of $0.1,0.3$, and 0.5 represent small, moderate, and large effect sizes, respectively.

\section{Results}

Out of a total of 486 stroke survivors prescreened for eligibility, 19 met the study criteria and agreed to participate (Table 1). The demographic characteristics were quite similar among the treatment groups. Of the 19 participants, 10 subjects with severe UE disability (median 14.6 of FMA-UE function scores) received rPMS after median 9.2 days from stroke onset, while 9 subjects (median 19.0) received standard care after 5.8 days $(Z=2.06, p=0.039)$, showing significantly different time periods to treatment between the two groups. The durations of treatment from baseline-pretreatment to follow-up estimation were median 7.8 and 16.5 sessions, respectively $(Z=2.12, p=0.034)$, demonstrating significantly shorter duration of the rPMS group relative to the SC group.

\subsection{Fugl-Meyer assessment outcomes}

FMA-UE function before treatment was not different $(p=0.97)$, and FMA-UE function after treatment also did not differ between the two groups $(p=0.90)$. At the same time, however, treatment durations were significantly different (average 7.8 sessions for rPMS group; 16.5 for SC group: $Z=2.12, p=0.034$ ). Therefore, the magnitude of increases in UE function was adjusted by the progress rate. The progress rate of FMA-UE scores differed significantly between groups, i.e., 2.65 for the rPMS group and 1.10 for the 


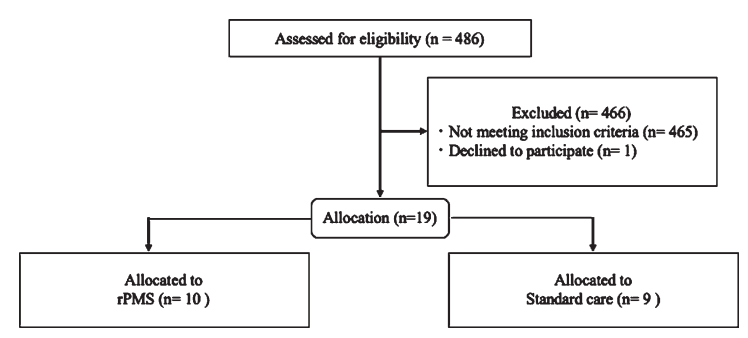

Fig. 1. Participant flow diagram.

SC group (U test; $U=8.5, z=2.982, p=0.003$ ). The rPMS group demonstrated large effect size $(r=0.68)$ on FMA-UE, suggesting high clinical significance in FMA-UE.

\subsection{Wolf motor function test}

Baseline WMFT-FAS scores were not significantly different $(p=0.86)$. Also, no differences, only trends, were observed in the magnitude of changes in WMFT-FAS scores between groups after treatment $(p=0.09, r=0.39)$. However, the progress rate of WMFT-FAS scores was significantly different $(U=9.0, Z=2.60, p=0.009)$. The rPMS group demonstrated large effect size on WMFT $(r=0.60)$, suggesting high clinical significance in WMFT-FAS.

\subsection{Box and block test}

Baseline BBT scores were not significantly different between the groups $(p=0.31)$. No significant differences were observed in the magnitude of changes in BBT scores after treatment between groups $(p=0.31)$. Also, the progress rate of BBT scores was not significantly different (U test; $U=31.5, Z=0.83, p=0.41)$. However, NMES group demonstrated moderate effect size on BBT $(r=0.19)$, suggesting low clinical significance with BBT.

\subsection{Side effects}

There were no adverse events such as pain or discomfort.

\section{Discussion}

The present study suggested the beneficial effect of rPMS for severe UE paresis during early acute phase of stroke. So far, previous reports have investigated the beneficial effect of rPMS on spasticity, but not on UE function in chronic phase of stroke (mean 26 weeks from onset) (Krewer, Hartl, Muller, \& Koenig, 2014). Differences from our study were the stimulation protocol and time to treatment (9 days versus 26 weeks). At the very least, it may depend on the stimulation protocol and treatment time as well as selection of stimulated muscles whether the rPMS would be effective for motor recovery after stroke.

In the present study, the two groups of rPMS and $\mathrm{SC}$ were significantly different in terms of time to treatment and treatment duration. The rPMS group had significantly more delay in time to treatment than the SC group. Given that the greatest gain of recovery tends to occur immediately after stroke, with a slower pace over time (Kwakkel et al., 2003, 2006), it is natural that the SC group should be at an advantage

Table 1

Participant demographics $(n=19)$

\begin{tabular}{lccc}
\hline Intervention & $\mathrm{SC}$ & rP.MS + SC & Statistics \\
\hline Participants & 9 & 10 & $\mathrm{Z}=1.47, p=0.14$ \\
Age (mean \pm SD) & $72.3 \pm 10.7(56-90)$ & $64.3 \pm 13.1(48-86)$ & \\
Sex female (\%) & $4(44.4)$ & $2(20)$ & \\
Type of stroke & 8 ischemia 1 hemorrhage & 8 ischemia 2 hemorrhage & $\mathrm{Z}=2.06, p=0.039, r=0.47$ \\
Time from onset to treatment, days & $5.8 \pm 2.2(2-9)$ & $92 \pm 4.4(3-18)$ & $\mathrm{Z}=0.04, p=0.97, r=0.09$ \\
Baseline FMA-UE scores (0-66) & $19.0 \pm 11.9(9-46)$ & $14.6 \pm 14.8(0-44)$ & $\mathrm{Z}=0.12, p=0.90, r=0.03$ \\
Follow-up FMA-UE scores & $36.5 \pm 17.3(15-63)$ & $38.3 \pm 15.5(20-62)$ & $\mathrm{Z}=0.18, p=0.86, r=0.04$ \\
Baseline WMFT-FAS scores (0-75) & $18.2 \pm 6.5(5-25)$ & $18.7 \pm 16.6(0-44)$ & $\mathrm{Z}=1.69, p=0.09, r=0.39$ \\
Follow-up WMFT-FAS scores & $30.4 \pm 6.7(23-41)$ & $40.8 \pm 15.5(22-70)$ & $\mathrm{Z}=1.01, p=0.31, r=0.23$ \\
Baseline BBT (number of blocks/min.) & $3.4 \pm 7.4(0-21)$ & $6.9 \pm 10.1(0-28)$ & $\mathrm{Z}=1.02, p=0.31, r=0.23$ \\
Follow-up BBT & $6.0 \pm 11.7(0-31)$ & $15.0 \pm 18.3(0-41)$ & $\mathrm{Z}=2.12,: p=0.034, r=0.49$ \\
Duration of treatment, days & $16.5 \pm 9.6(8-32)$ & $7.8 \pm 3.5(3-14)$ & $\mathrm{Z}=2.98,{ }^{*} p=0.003,{ }^{* *} r=0.68$ \\
Progress rate (FMA-UE) & $1.10 \pm 0.79$ & $2.65 \pm 1.51$ & $\mathrm{Z}=2.60,{ }^{*} p=0.009,{ }^{* *} r=0.60$ \\
Progress rate (WMFT-FAS) & $0.91 \pm 0.65$ & $3.0 \pm 2.50$ & $\mathrm{Z}=0.83, p=0.41, r=0.19$ \\
Progress rate (BBT) & $0.37 \pm 0.68$ & $1.41 \pm 1.99$ &
\end{tabular}

BBT: box and block test; FAS: functional ability scale; FMA-UE: Fugl-Meyer motor assessment scale for upper extremity; rPMS: peripheral magnetic stimulation; SC: standard care group; WMFT: Wolf motor function test. ${ }^{*} p<0.01 ;{ }^{* *} r \leq 0.5$. 
in terms of motor recovery. Nevertheless, the rPMS group showed significant recovery of UE paresis as compared to the SC group. In addition, age difference was negligible and was not enough to explain the significant difference of the progress rate. Together, we claim that favorable effect on UE function might be mainly attributable to rPMS treatment.

The pros and cons of rPMS have been discussed (Beaulieu \& Schneider, 2015). Advantages of rPMS over NMES include no pain, deeper penetration, generation of higher muscle torque, and applicability to children. In contrast, disadvantages involve overheating of the coil and a larger area stimulated with increased intensity. So far, however, no recommendations have been provided regarding the parameters of rPMS application, such as coil design, duty cycle, duration, frequency and intensity. As for the duty cycle, intermittent stimulation or continuous stimulation has been applied in previous trials. In particular, cyclic muscle contraction/relaxation arising from intermittent stimulation generates massive proprioceptive flows to the affected hemisphere mediated by thalamo-cortical and cotrico-cortical fibers (Flamand \& Schneider, 2014). In other words, intermittent rPMS might induce cortical neuroplastic changes in conjunction with motor recovery. In this regard, a previous PET activation study (Struppler et al., 2007) delineated the reorganization of the motor map induced by rPMS. The authors demonstrated that regional cerebral blood flow (rCBF) increases in the superior posterior parietal cortex, and the premotor cortex was associated with an improvement of spasticity in the paretic arm following rPMS treatment. In addition, there is increasing evidence that motor recovery after stroke is associated with reorganization of damaged brain (Grefkes \& Fink, 2014; Nudo, Wise, SiFuentes, \& Milliken, 1996). Specifically, Nudo and colleagues demonstrated that, during intensive rehabilitative training, monkeys recovered from UE paresis after infarction in association with enlargement of the motor map representing the disabled forearm (Nudo et al., 1996). Similarly, some studies have proposed the possible neural mechanism underlying UE function recovery mediated by peripheral stimulation, such as NMES. Our near-infrared spectroscopy study suggested the association of the ipsilateral sensorimotor cortex with the improvement of UE function by EMG-triggered NMES in chronic phase of stroke patients (Hara et al., 2013). Another neuroimaging study demonstrated the activation of the contralateral sensorimotor cortex by electrically stimulating the wrist extensor and flexor muscles
(Blickenstorfer et al., 2009). In addition, an ischemic model study revealed that electrical stimulation of the disabled forepaw at early stage of ischemia improved function while reducing the infarct volume (Burnett et al., 2006). These findings might support the superiority of peripheral stimulation for affected UE at early acute phase of stroke. Given that peripheral stimulation can induce neuroplastic changes associated with motor recovery, it is plausible that rPMS can help improve UE function as well as NMES. In fact, we showed that rPMS, particularly when starting with early acute phase of stroke, was effective for UE function recovery. Furthermore, our results suggested that, in comparison, lower dose and shorter duration of intervention ameliorate UE disability during early acute phase of stroke.

\subsection{Limitations}

This study includes several limitations. Because of the small sample size, the results should be interpreted with caution. We need to accumulate a larger sample size with sufficient power to yield definitive statistical results. Further study would be required to find more optimal stimulation parameters for accelerating the beneficial effects of rPMS on motor recovery. Further study would also be required to disclose the relationship between dose and motor recovery during acute phase of stroke. In addition, some participants demonstrated an accelerated progress rate, and inter-subject difference in the magnitude of motor recovery existed not only in the rPMS intervention group but also in the SC group. In the future, we need to explore how the difference in magnitude of improvement would be achieved. Finally, eligibility criteria may be required to decide which of the alternative interventions, rPMS and NMES, is more effective for improving upper extremity disability after stroke.

\section{Conclusions}

This is the first report to describe beneficial effects of rPMS on severe UE dysfunction during early acute phase of stroke when applying rPMS to the whole UE. These findings would contribute to the justification for specific treatment parameters to maximize severe UE recovery after stroke. In addition, we strongly desire that our results might lead to the establishment of a definitive approach to the improvement of severe UE paresis immediately after stroke onset. 


\section{Acknowledgments}

The authors wish to thank their co-medical staff in the acute rehabilitation care unit for their cooperation. This work was partially supported by a grant from Grants-in-Aid for Scientific Research, Japan Society for the Promotion of Science (JSPS) to S.O (19K11355).

\section{Conflict of interest}

\section{None to report.}

\section{References}

Barker, A. T. (1991). An introduction to the basic principles of magnetic nerve stimulation. J Clin Neurophysiol, 8(1), 26-37. doi:10.1097/00004691-199101000-00005

Beaulieu, L. D., \& Schneider, C. (2013). Effects of repetitive peripheral magnetic stimulation on normal or impaired motor control. A review. Neurophysiol Clin, 43(4), 251-260. doi:10.1016/j.neucli.2013.05.003

Beaulieu, L. D., \& Schneider, C. (2015). Repetitive peripheral magnetic stimulation to reduce pain or improve sensorimotor impairments: A literature review on parameters of application and afferents recruitment. Neurophysiol Clin, 45(3), 223-237. doi:10.1016/j.neucli.2015.08.002

Blickenstorfer, A., Kleiser, R., Keller, T., Keisker, B., Meyer, M., Riener, R., \& Kollias, S. (2009). Cortical and subcortical correlates of functional electrical stimulation of wrist extensor and flexor muscles revealed by fMRI. Hum Brain Mapp, 30(3), 963-975. doi:10.1002/hbm.20559

Burnett, M. G., Shimazu, T., Szabados, T., Muramatsu, H., Detre, J. A., \& Greenberg, J. H. (2006). Electrical forepaw stimulation during reversible forebrain ischemia decreases infarct volume. Stroke, 37(5), 1327-1331. doi:10.1161/01.STR.0000217305.82123.d8

Carda, S., Biasiucci, A., Maesani, A., Ionta, S., Moncharmont, J., Clarke, S., ...\& Millan, J. D. R. (2017). Electrically Assisted Movement Therapy in Chronic Stroke Patients With Severe Upper Limb Paresis: A Pilot, Single-Blind, Randomized Crossover Study. Arch Phys Med Rehabil, 98(8), 1628-1635 e1622. doi:10.1016/j.apmr.2017.02.020

Cauraugh, J., Light, K., Kim, S., Thigpen, M., \& Behrman, A. (2000). Chronic motor dysfunction after stroke: recovering wrist and finger extension by electromyographytriggered neuromuscular stimulation. Stroke, 31(6), 13601364. doi:10.1161/01.str.31.6.1360

Chae, J., Bethoux, F., Bohine, T., Dobos, L., Davis, T., \& Friedl, A. (1998). Neuromuscular stimulation for upper extremity motor and functional recovery in acute hemiplegia. Stroke, 29(5), 975-979. doi:10.1161/01.str.29.5.975

Desrosiers, J., Bravo, G., Hebert, R., Dutil, E., \& Mercier, L. (1994). Validation of the Box and Block Test as a measure of dexterity of elderly people: reliability, validity, and norms studies. Arch Phys Med Rehabil, 75(7), 751-755.
Flamand, V. H., \& Schneider, C. (2014). Noninvasive and painless magnetic stimulation of nerves improved brain motor function and mobility in a cerebral palsy case. Arch Phys Med Rehabil, 95(10), 1984-1990. doi:10.1016/j.apmr.2014.05.014

Francisco, G., Chae, J., Chawla, H., Kirshblum, S., Zorowitz, R., Lewis, G., \& Pang, S. (1998). Electromyogram-triggered neuromuscular stimulation for improving the arm function of acute stroke survivors: a randomized pilot study. Arch Phys Med Rehabil, 79(5), 570-575. doi:10.1016/s0003-9993(98)90 074-0

Fugl-Meyer, A. R., Jaasko, L., Leyman, I., Olsson, S., \& Steglind, S. (1975). The post-stroke hemiplegic patient. 1. a method for evaluation of physical performance. Scand J Rehabil Med, 7(1), 13-31.

Grefkes, C., \& Fink, G. R. (2014). Connectivity-based approaches in stroke and recovery of function. Lancet Neurol, 13(2), 206216. doi: 10.1016/S1474-4422(13)70264-3

Hara, Y., Obayashi, S., Tsujiuchi, K., \& Muraoka, Y. (2013). The effects of electromyography-controlled functional electrical stimulation on upper extremity function and cortical perfusion in stroke patients. Clin Neurophysiol, 124(10), 2008-2015. doi:10.1016/j.clinph.2013.03.030

Hsu, S. S., Hu, M. H., Wang, Y. H., Yip, P. K., Chiu, J. W., \& Hsieh, C. L. (2010). Dose-response relation between neuromuscular electrical stimulation and upper-extremity function in patients with stroke. Stroke, 41(4), 821-824. doi:10.1161/STROKEAHA.109.574160

Krewer, C., Hartl, S., Muller, F., \& Koenig, E. (2014). Effects of repetitive peripheral magnetic stimulation on upper-limb spasticity and impairment in patients with spastic hemiparesis: a randomized, double-blind, shamcontrolled study. Arch Phys Med Rehabil, 95(6), 1039-1047. doi:10.1016/j.apmr.2014.02.003

Kwakkel, G., Kollen, B., \& Twisk, J. (2006). Impact of time on improvement of outcome after stroke. Stroke, 37(9), 23482353. doi:10.1161/01.STR.0000238594.91938.1e

Kwakkel, G., Kollen, B. J., van der Grond, J., \& Prevo, A. J. (2003). Probability of regaining dexterity in the flaccid upper limb: impact of severity of paresis and time since onset in acute stroke. Stroke, 34(9), 2181-2186. doi:10.1161/01.STR.0000087172.16305.CD

Nudo, R. J., Wise, B. M., SiFuentes, F., \& Milliken, G. W. (1996). Neural substrates for the effects of rehabilitative training on motor recovery after ischemic infarct. Science, 272(5269), 1791-1794. doi:10.1126/science.272.5269.1791

Obayashi, S. (2020). Upper limb recovery in early acute phase stroke survivors by coupled EMG-triggered and cyclic neuromuscular electrical stimulation. NeuroRehabilitation, 46(3), 417-422.

Powell, J., Pandyan, A. D., Granat, M., Cameron, M., \& Stott, D. J. (1999). Electrical stimulation of wrist extensors in poststroke hemiplegia. Stroke, 30(7), 1384-1389. doi:10.1161/01.str.30.7.1384

Struppler, A., Angerer, B., Gundisch, C., \& Havel, P. (2004). Modulatory effect of repetitive peripheral magnetic stimulation on skeletal muscle tone in healthy subjects: stabilization of the elbow joint. Exp Brain Res, 157(1), 59-66. doi:10.1007/s00221-003-1817-6

Struppler, A., Binkofski, F., Angerer, B., Bernhardt, M., Spiegel, S., Drzezga, A., \& Bartenstein, P. (2007). A frontoparietal network is mediating improvement of motor function related to repetitive peripheral magnetic stimulation: A 
PET-H2O15 study. Neuroimage, 36 Suppl 2, T174-186. doi:10.1016/j.neuroimage.2007.03.033

Struppler, A., Havel, P., \& Muller-Barna, P. (2003). Facilitation of skilled finger movements by repetitive peripheral magnetic stimulation (RPMS) - a new approach in central paresis. NeuroRehabilitation, 18(1), 69-82.

Whitall, J. (2004). Stroke rehabilitation research: time to answer more specific questions? Neurorehabil Neural Repair, 18(1), 3-8; author reply 9-11. doi:10.1177/0888439003262876

Wilson, R. D., Page, S. J., Delahanty, M., Knutson, J. S., Gunzler, D. D., Sheffler, L. R., \& Chae, J. (2016). Upper-Limb
Recovery After Stroke: A Randomized Controlled Trial Comparing EMG-Triggered, Cyclic, and Sensory Electrical Stimulation. Neurorehabil Neural Repair, 30(10), 978-987. doi:10.1177/1545968316650278

Wolf, S. L., Catlin, P. A., Ellis, M., Archer, A. L., Morgan, B., \& Piacentino, A. (2001). Assessing Wolf motor function test as outcome measure for research in patients after stroke. Stroke, 32(7), 1635-1639. doi:10.1161/01.str.32.7.1635 